\title{
Efficacy of ginkgo biloba extract as augmentation of venlafaxine in treating post-stroke depression
}

This article was published in the following Dove Press journal:

Neuropsychiatric Disease and Treatment

\author{
Zi-Hong Liang' \\ Yan-Bo Jia ${ }^{2}$ \\ Mei-Ling Wang' \\ $\mathrm{Zi}-\mathrm{Ru} \mathrm{Li}^{\prime}$ \\ Min $\mathrm{Li}^{\mathrm{I}}$ \\ Yong-Li Yun' \\ Run-Xiu Zhu' \\ 'Department of Neurology, Inner \\ Mongolia Autonomous Region People's \\ Hospital, Huhhot, Inner Mongolia, \\ People's Republic of China; ${ }^{2}$ Department \\ of Orthopaedics, The Second Affiliated \\ Hospital of Inner Mongolia Medical \\ University, Huhhot, Inner Mongolia, \\ People's Republic of China
}

Correspondence: Run-Xiu Zhu Department of Neurology, Inner Mongolia Autonomous Region People's Hospital, No. 20 Zhaowuda Road,

Huhhot, Inner Mongolia 010017, People's Republic of China

$\mathrm{Tel}+86047 \mathrm{I} 3283999$

Fax +8604713283999

Email xcinhah-2I@foxmail.com
Background: Post-stroke depression (PSD) is one of the most common psychiatric diseases afflicting stroke survivors. This study was conducted to assess the efficacy of ginkgo biloba extract as augmentation of venlafaxine in treating PSD.

Methods: The included PSD patients were randomly assigned into the experiment group (receiving ginkgo biloba extract plus venlafaxine) and control group (receiving venlafaxine alone). The treatment was continued for eight weeks. The Hamilton Depression Rating Scale (HDRS) and the Self-rating Depression Scale (SDS) were used to assess the depressive symptoms. The National Institutes of Health Stroke Scale (NIHSS) was used to assess the neurological defect, and the Activities of Daily Living (ADL) was used to assess recovery of abilities of patients after stroke. Meanwhile, the levels of serum 5-hydroxytryptamine (5HT) and brain-derived neurotrophic factor (BDNF) were measured before and after treatment. The dose of venlafaxine used and adverse events were also recorded.

Results: Each group had 40 PSD patients. After treatment, the depressive symptoms, neurological defect and living function were significantly improved in both groups. But the patients receiving ginkgo biloba extract plus venlafaxine had the significantly lower average HDRS score $(p=0.0008)$, SDS score $(p<0.00001)$, NIHSS score $(p=0.00001)$, and higher average ADL score $(p=0.0005)$. Meanwhile, compared to the control group, patients in the experiment group had the significantly higher 5-HT $(p<0.00001)$ level and BDNF level $(p<0.00001)$, needed lower dose of venlafaxine $(p=0.007)$, and experienced fewer adverse events.

Conclusion: These results demonstrated that the ginkgo biloba extract was a good augmentation of venlafaxine in treating PSD and should be further investigated.

Keywords: ginkgo biloba extract, venlafaxine, post-stroke depression, PSD

\section{Introduction}

Post-stroke depression (PSD) is one of the most common psychiatric diseases afflicting stroke survivors. ${ }^{1}$ It is estimated that about $30 \%$ of the stroke survivors will experience an early or later onset of depression. ${ }^{2,3}$ In 2013, researchers from China reported that the number of stroke survivors was 7.5 million, and there were 2.5 million new stroke cases each year; it indicated that there were approximately three million PSD patients. ${ }^{4}$ PSD has deleterious effects on the rehabilitation and recovery process, and could increase disability and mortality. ${ }^{5}$ Moreover, PSD might affect the patients' acceptability and the effectiveness of therapy. ${ }^{6}$ However, a large number of PSD patients are still unrecognized and/or undertreated.

One important reason is that there are no objective methods to diagnose PSD or depression is viewed as a normal emotional reaction to stroke. ${ }^{7,8}$ Another important 
reason is that the clinicians concern about the safety of multi-pharmacological interventions in elderly stroke patients. Nowadays, there is a consensus that stroke patients with depression should be treated immediately once identified. But, the most appropriate management strategy for treating PSD is still not identified. Hackett and Anderson used cognitive-behavioral therapy to treat elderly PSD patients and observed some improvement. ${ }^{9} \mathrm{~A}$ systematic review reported that pharmacotherapy could improve the depressive symptoms of PSD patients. ${ }^{10}$

Many Chinese medicines are often used as augmentations of antidepressants in treating PSD patients. ${ }^{11,12}$ Wang reported that Wuling capsule could increase the peripheral levels of BDNF in PSD patients. ${ }^{11}$ Another study found that the combined application of Xiaoyao pill and fluoxetine could improve the depressive symptoms of PSD patients. ${ }^{12}$ Recently, some studies suggested that ginkgo biloba extract could improve the efficacy of antidepressants in treating depression. ${ }^{13,14}$ However, the action mechanism of ginkgo biloba extract in depression is still unclear, which might be closely related to its neuroprotective actions. ${ }^{15,16}$ In our previous studies, we found that ginkgo biloba extract could affect nucleotide metabolism and protein biosynthesis to enhance cell proliferation, and glutamate and aspartate metabolism played important roles in the antidepressant effects of ginkgo biloba extract. ${ }^{17-19}$ These two pathways were associated with neurotransmitter metabolism. Previous study reported that the plasma levels of glutamate after stroke were associated with the development of PSD. ${ }^{20}$ Therefore, we conducted this randomized clinical trial to further study the efficacy of ginkgo biloba extract as augmentation of venlafaxine in treating post-stroke depression.

\section{Methods}

\section{Patients' recruitment}

The protocol of our study was approved by the Ethical Committee of Inner Mongolia People's Hospital (Huhhot, Inner Mongolia, China). This study was conducted in accordance with the Declaration of Helsinki. The candidates were informed the purpose of our study, and the included patients provided the written informed consents before randomization. The patients were recruited from the Department of Neurology, Inner Mongolia Autonomous Region People's Hospital (Huhhot, Inner Mongolia, China). The diagnostic criteria for ischemic stroke that was revised by the fourth National Conference on
Cerebrovascular Diseases were used to diagnose stroke, and the stroke was also confirmed by magnetic resonance imaging or computed tomography. Meanwhile, the Hamilton Depression Rating Scale (HDRS) was used to diagnose depression in stroke patients. ${ }^{21}$ The stroke patients with HDRS scores of greater than 17 were diagnosed with depression and included. ${ }^{22}$ Patients were excluded if they were aged 80 years and older, had active suicidal ideation, acute illness, any other neuropsychiatric disorders, any anti-depressive therapy or illicit drug use. All PSD patients were from the same place and of the same ethnicity.

\section{Treatment methods}

The included PSD patients were randomly assigned to the control group and experiment group at a ratio of 1:1. Patients in both groups were received venlafaxine $75 \mathrm{mg} /$ day; if needed, the dose could increase to a maximum of 225 $\mathrm{mg} /$ day within 4 days. At the end of the trial, the dose was gradually reduced. The ginkgo biloba extract ( $40 \mathrm{mg} / \mathrm{time}, 3$ times a day) was only given to the patients in the experiment group. The treatment was continued for 8 weeks. Patients were blinded to the treatment methods. The interviewer who was blinded to the treatment methods measured the HDRS score, National Institutes of Health Stroke Scale (NIHSS) score, and Activities of Daily Living (ADL) score at baseline, week 4, and week 8 . Patients also should provide the Self-rating Depression Scale (SDS) scores at baseline, week 4, and week 8. Meanwhile, the levels of serum 5-hydroxytryptamine (5-HT) and brain-derived neurotrophic factor (BDNF) were measured before and after treatment.

\section{Efficacy assessment}

The HDRS and SDS scores were used to assess the depressive symptoms. The higher scores suggested the more severe depressive symptoms. The NIHSS score was used to assess the neurological defect, and the ADL score was used to assess the recovery of abilities of patients following strokes. The higher NIHSS score indicated the more severe neurological defect, and the lower ADL score indicated the worse living function. Meanwhile, to assess whether the ginkgo biloba extract could reduce the dose of venlafaxine, the dose of venlafaxine used in both groups was collected. To assess the acceptability of these two treatment methods, the adverse events were also recorded. 


\section{Statistical analysis}

Data were displayed as mean \pm standard deviation, and all data were tested for normal distribution before conducting analysis. The Student's $t$-test, Chi-square test, and nonparametric Mann-Whitney U test were used when appropriate. Analyses for HDRS score, SDS score, NIHSS score, and ADL score were conducted using the repeated measures analysis of variance (ANOVA). Analyses for 5HT and BDNF were conducted using Student's $t$-test. The SPSS19.0 was used for statistical analysis, and the statistical significance was set to $p<0.05$.

\section{Results}

\section{Included patients}

At first, there were 93 PSD patients met the inclusion/ exclusion criteria. But, there were six patients refusing to receive ginkgo biloba extract, four patients refusing the randomization, and three patients refusing to participate. Thus, these patients were excluded, and only 80 PSD patients were included. According to the observed posttreatment improvement from the pilot study, 74 patients were needed to achieve an $80 \%$ power at a given level of significance $(p<0.05)$. Thus, a total of 80 patients here could achieve an $85 \%$ power for detection of a clinically meaningful difference between the two different groups. The detailed information of these patients is described in Table 1.

\section{Adverse events}

During the whole treatment period, the following adverse events appeared in the control group: abdominal distension $(n=3)$, dizziness $(n=4)$, nausea $(n=2)$, headache $(n=5)$,

Table I Clinical detail of patients in the experiment and control group

\begin{tabular}{|l|l|l|l|}
\hline Variables & $\begin{array}{l}\text { Experiment } \\
\text { group }\end{array}$ & $\begin{array}{l}\text { Control } \\
\text { group }\end{array}$ & $p$-value \\
\hline $\mathrm{n}$ & 40 & 40 & - \\
Female/male & $18 / 22$ & $21 / 19$ & $0.51^{\mathrm{b}}$ \\
Age (years) & $60.86(8.63)$ & $60.52(8.68)$ & $0.86^{\mathrm{a}}$ \\
Education (years) & $6.92(3.05)$ & $7.20(2.96)$ & $0.68^{\mathrm{a}}$ \\
Body mass index $\left(\mathrm{kg} / \mathrm{m}^{2}\right)$ & $23.02(3.57)$ & $23.16(3.27)$ & $0.86^{\mathrm{a}}$ \\
Smoking (yes $/ \mathrm{no})$ & $15 / 25$ & $1 \mathrm{I} / 29$ & $0.35^{\mathrm{b}}$ \\
Duration of stroke & $22.59(7.3)$ & $21.98(7.94)$ & $0.72^{\mathrm{a}}$ \\
(months) & & & \\
Duration of depression & $11.31(4.24)$ & $11.03(4.77)$ & $0.78^{\mathrm{a}}$ \\
(months) & & & \\
\hline
\end{tabular}

Note: ${ }^{\mathrm{a} T w o-t a i l e d ~ S t u d e n t ' s ~} t$-test; ${ }^{\mathrm{b}} \mathrm{Chi}$-square test. drowsiness $(n=6)$, insomnia $(n=4)$, and fatigue $(n=3)$; the following adverse events appeared in the experiment group: constipation $(\mathrm{n}=3)$, dizziness $(\mathrm{n}=2)$, nausea $(\mathrm{n}=1)$, headache $(n=2)$, drowsiness $(n=3)$, and insomnia $(n=1)$. Compared to the control group, the experiment group had less adverse events. These adverse events were mild and disappear soon, and no serious adverse events appeared in both groups. Meanwhile, compared to PSD patients in the control group, PSD patients in the experiment group needed significantly lower average dose of venlafaxine $(6930 \mathrm{mg}$ vs $6037 \mathrm{mg}$ for each patient, $p=0.007)$.

\section{Depressive symptoms}

As shown in Figure 1, the average HDRS scores decreased over time in both groups. The significant effect of time $(p<0.00001)$ was identified by the results of repeated measures ANOVA, indicating that both treatment methods could significantly improve the depressive symptoms of PSD patients. Meanwhile, there was also a significant effect of group $\times$ time interaction $(p=0.01)$, which indicated that the efficacy of these two treatment methods in improving the depressive symptoms was significantly different. At week 4 and week 8, the average HDRS score was significantly lower in the experiment group than in the control group ( $p=0.001, p=0.0008$ ). The repeated measures ANOVA of SDS scores also showed similar results.

\section{Neurological and living function}

As shown in Figure 2, the average NIHSS scores decreased over time in both groups. The significant effect of time ( $p=0.003$ ) was identified by the results of repeated measures ANOVA, indicating that both treatment methods could significantly improve the neurological defect of PSD patients. Meanwhile, there was also a significant effect of group $\times$ time interaction $(p=0.01)$, which indicated that the efficacy of these two treatment methods in improving the neurological defect was significantly different. At week 4 and week 8 , the average NIHSS score was significantly lower in the experiment group than in the control group ( $p=0.00006, p=0.00001$ ). The repeated measures ANOVA of ADL scores also showed similar results, indicating the significantly different efficacy of these two treatment methods in improving the living function.

\section{5-HT and BDNF}

As shown in Figure 3, both the 5-HT levels and BDNF levels were similar between the two groups. After treatment, the 

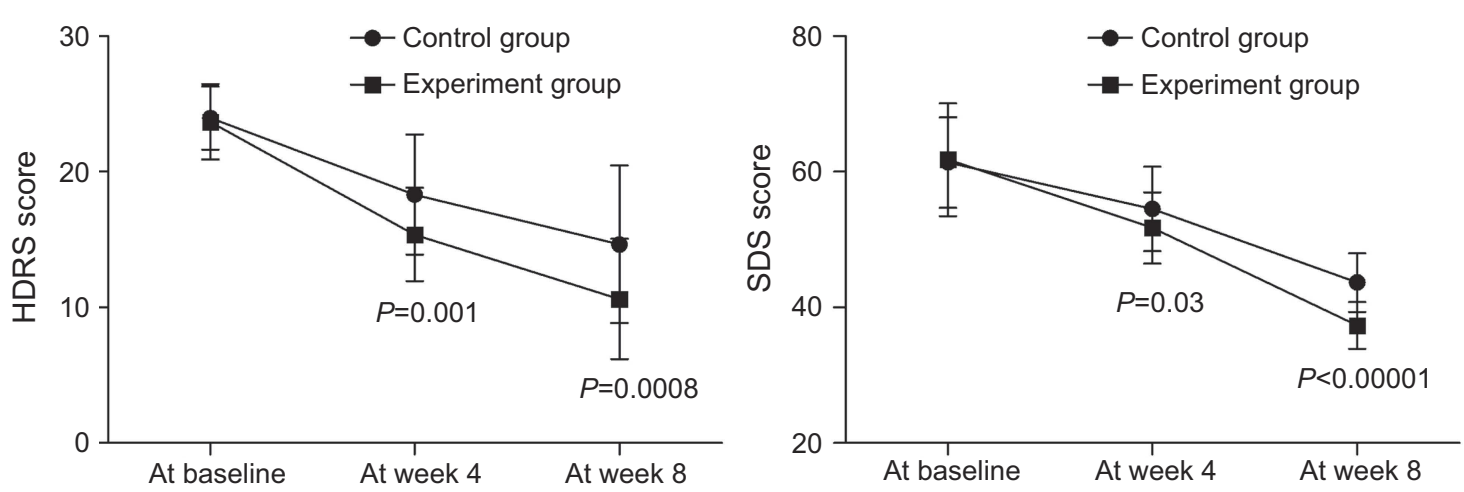

Figure I The Hamilton Depression Rating Scale (HDRS) and the Self-rating Depression Scale (SDS) scores in both groups before and after treatment.
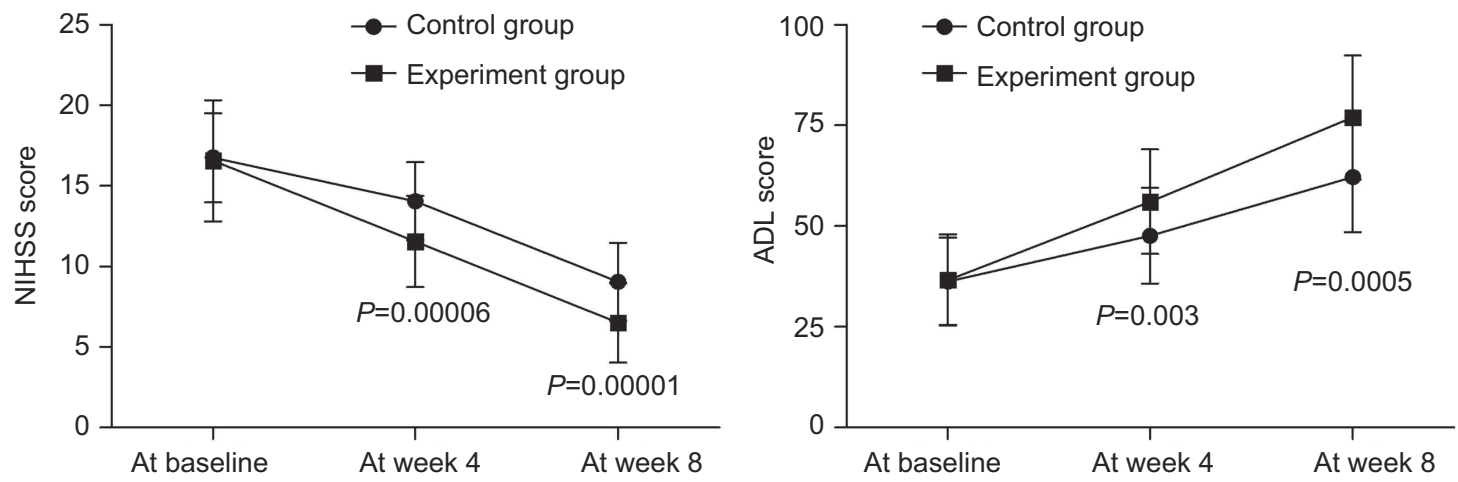

Figure 2 The National Institutes of Health Stroke Scale (NIHSS) and the Activities of Daily Living (ADL) scores in both groups before and after treatment.
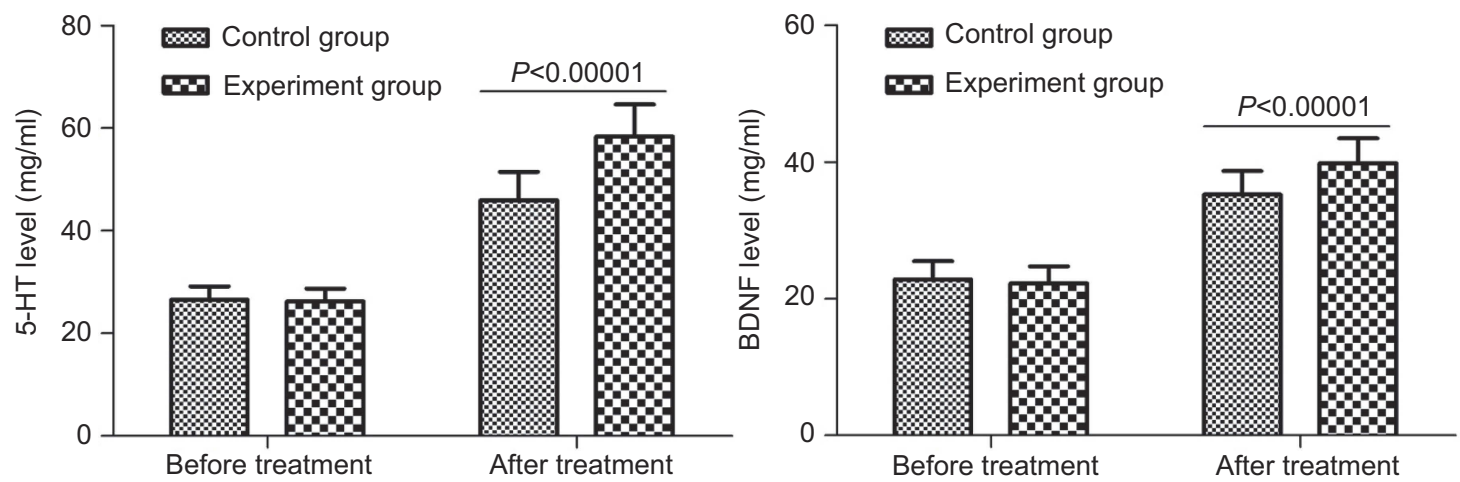

Figure 3 Serum 5-hydroxytryptamine (5-HT) and brain-derived neurotrophic factor (BDNF) levels in both groups before and after treatment.

average 5-HT level was significantly increased from 26.57 (2.57) $\mathrm{mg} / \mathrm{mL}$ to $45.98(5.51) \mathrm{mg} / \mathrm{mL}$ in the control group $(p<0.00001)$, and from $26.24(2.56) \mathrm{mg} / \mathrm{mL}$ to 58.34 (6.28) $\mathrm{mg} / \mathrm{ml}$ in the experiment group $(p<0.00001)$. But, the average 5-HT level after treatment was significantly higher in the experiment group than in the control group $(p<0.00001)$. Meanwhile, after treatment, the average BDNF level was significantly increased from $22.87(2.64) \mathrm{mg} / \mathrm{mL}$ to 35.26 (3.45) $\mathrm{mg} / \mathrm{mL}$ in the control group $(p<0.00001)$, and from
$22.25(2.55) \mathrm{mg} / \mathrm{mL}$ to $39.87(3.68) \mathrm{mg} / \mathrm{ml}$ in the experiment group $(p<0.00001)$. But, the average BDNF level after treatment was significantly higher in the experiment group than in the control group $(p<0.00001)$.

\section{Discussion}

Depression is a debilitating mental disorder with unclear pathogenesis and could cause a substantial impact on individuals and society. ${ }^{23,24}$ Saxena et al reported that the 
improvement in depressive symptoms in PSD patients might be helpful for their recovery in activities of daily living. ${ }^{25}$ In this study, we found that the two treatment methods could be effective in treating PSD. But the combined application of ginkgo biloba extract and venlafaxine yielded the significantly lower average HDRS score, SDS score, NIHSS score, and higher average ADL score. Furthermore, the consumed dose of venlafaxine and the incidence of adverse events were lower in the experiment group compared to the control group. These results suggested that it might be a good choice to use ginkgo biloba extract as augmentation of venlafaxine in treating PSD.

BDNF is widely found in many brain regions, such as hippocampus and cerebral cortex. It could promote the differentiation and regeneration of damaged neurons and affect the release of neurotransmitters and trophic factors. ${ }^{26}$ Sato et al reported that BNDF played a critical role in protecting and promoting the regeneration of nerve fibers in a stroke model of monkeys. ${ }^{27}$ Previous studies have found lower serum BDNF levels in patients with depression. ${ }^{28,29}$ A systematic review reported that the antidepressant treatment might be effective in increasing the serum BDNF levels of depressed patients. ${ }^{30}$ Our previous study also found that BDNF was a key molecule in the process of venlafaxine exerting antidepressant effects. ${ }^{31}$ Here, we found that compared to venlafaxine alone, the combined application of ginkgo biloba extract and venlafaxine could yield the significantly higher BDNF levels in PSD patients. Therefore, the ginkgo biloba extract and venlafaxine might have a synergistic effect in increasing the BDNF levels in PSD patients.

5-HT is one of the important excitatory neurotransmitters in the central nervous system and distributes in various brain regions. Previous studies have confirmed that the pathogenesis of depression was associated with 5-HT disturbance. $^{32,33}$ In the normal human brain, there is a close relationship between BDNF and 5-HT. The BDNF can enhance the signal transduction of 5-HT, and 5-HT can stimulate the expression of BDNF. But, this relationship is interrupted when the brain undergoes ischemia and hypoxia, which could result in the low expression of BDNF and 5 -HT. ${ }^{34}$ In this study, we found that both serum BDNF levels and serum 5-HT levels were significantly higher in the experiment group than in the control group. These results indicated that the ginkgo biloba extract could enhance the antidepressant effect of venlafaxine.
Some limitations of this study should be mentioned here. First, the number of PSD patients was relatively small; then future studies were needed to verify our conclusion. Second, all PSD patients were from the same place, which might limit the applicability of our results. ${ }^{35,36}$ Third, the long-term efficacy of the combined application of ginkgo biloba extract and venlafaxine was not be assessed here. Finally, we only measured the BDNF levels and 5-HT levels in serum, future studies should measure their levels in cerebrospinal fluid to further ensure the action mechanisms of ginkgo biloba extract and venlafaxine.

In conclusion, we found that the ginkgo biloba extract was a good augmentation of venlafaxine in treating PSD. Meanwhile, the addition of ginkgo biloba extract could significantly reduce the dose of venlafaxine used, which was very important for elderly patients. Also, there were fewer adverse events in the experiment group than in the control group. Therefore, the clinical applicability of ginkgo biloba extract plus venlafaxine showed greater promise and should be further investigated by future studies with larger sample sizes.

\section{Acknowledgment}

This project is funded by the Health Family Planning Research Program of Inner Mongolia Autonomous Region (Grant No. 201703005), the Natural Science Foundation of Inner Mongolia Autonomous Region of China (Grant No. 2016MS0884), and the Foundation Project of the Inner Mongolia Autonomous Region People's Hospital (Grant No. 201551).

\section{Disclosure}

The authors declare no financial or other conflicts of interest in this work.

\section{References}

1. Robinson RG, Spalletta G. Poststroke depression: a review. Can J Psychiatry. 2010;55:341-349. doi:10.1177/070674371005500602

2. Whyte EM, Mulsant BH, Vanderbilt J, et al. Depression after stroke: a prospective epidemiological study. J Am Geriatr Soc. 2004;52:774778. doi:10.1111/j.1532-5415.2004.52217.x

3. Meader N, Moe-Byrne T, Llewellyn A, Mitchell AJ. Screening for poststroke major depression: a meta-analysis of diagnostic validity studies. J Neurol Neurosurg Psychiatry. 2014;85(2):198-206. doi:10.1136/jnnp-2012-304194

4. Tu WJ, Dong X, Zhao SJ, et al. Prognostic value of plasma neuroendocrine biomarkers in patients with acute ischaemic stroke. $J$ Neuroendocrinol. 2013;25:771-778. doi:10.1111/jne.12052 
5. Zhan Y, Yang YT, You HM, et al. Plasma-based proteomics reveals lipid metabolic and immunoregulatory dysregulation in post-stroke depression. Eur Psychiatry. 2014;29(5):307-315. doi:10.1016/j.eurpsy.2014.03.004

6. Srivastava A, Taly AB, Gupta A, Murali T. Post-stroke depression: prevalence and relationship with disability in chronic stroke survivors. Ann Indian Acad Neurol. 2010;13:123-127. doi:10.4103/0972-2327.64643

7. House A, Dennis M, Hawton K, Warlow C. Methods of identifying mood disorders in stroke patients: experience in the Oxfordshire Community Stroke Project. Age Ageing. 1989;18(6):371-379. doi:10.1093/ageing/18.6.371

8. Liu Z, Cai Y, He J. High serum levels of 8-OHdG are an independent predictor of post-stroke depression in Chinese stroke survivors. Neuropsychiatr Dis Treat. 2018;14:587-596. doi:10.2147/NDT.S155144

9. Hackett ML, Anderson CS. Treatment options for post-stroke depression in the elderly. Aging Health. 2005;1(1):95-105. doi:10.2217/ 1745509X.1.1.95

10. Hackett ML, Anderson CS, Hose AO. Management of depression after stroke: a systematic review of pharmacological therapies. Stroke. 2005;36(5):1098-1103. doi:10.1161/01.STR.0000162391.27991.9d

11. Wang J. To investigate the depression after Wuling capsule on ischemic stroke patients serum Hcy, nerve cell factor, single amine neurotransmitter metabolism and the influence of the inflammatory response. Shaanxi J Trad Chin Med. 2018;39(10):1357-1359.

12. Zeng ML, Chen L, Li B, et al. Effects of xiaoyao pill combined with fluoxetine in treating post-stroke depression and its influence on the serum 5-serotonin level. Zhejiang J Integr Trad Chin West Med. 2018;28(12):997-999.

13. Ma YS. Therapeutic effect of Ginkgo biloba extract combined with SSRI drugs on depression in elderly patients with coronary heart disease. Chin Prac Med. 2012;7(19):49-50.

14. Wu HX, Ge MJ, Hao FL, Zhang X, Yuan LF. Therapeutic effect evaluation of Ginkgo biloba extract united with fluoxetine on acute cerebral infarction depression. Int J Psychiatry. 2016;43(3):474-476.

15. Ma S, Liu X, Xu Q, et al. Transport of ginkgolides with different lipophilicities based on an hCMEC/D3 cell monolayer as a bloodbrain barrier cell model. Life Sci. 2014;114(2):93-101. doi:10.1016/j. 1fs.2014.08.006

16. Maclennan KM, Darlington CL, Smith PF. The CNS effects of Ginkgo biloba extracts and ginkgolide B. Prog Neurobiol. 2002;67 (3):235-257.

17. Bai S, Zhang X, Chen Z, et al. Insight into the metabolic mechanism of Diterpene Ginkgolides on antidepressant effects for attenuating behavioural deficits compared with venlafaxine. Sci Rep. 2017;7 (1):9591. doi:10.1038/s41598-017-10391-1

18. Hu Q, Shen P, Bai S, et al. Metabolite-related antidepressant action of diterpene ginkgolides in the prefrontal cortex. Neuropsychiatr Dis Treat. 2018;14:999-1011. doi:10.2147/NDT.S161351

19. Liang Z, Bai S, Shen P, et al. GC-MS-based metabolomic study on the antidepressant-like effects of diterpene ginkgolides in mouse hippocampus. Behav Brain Res. 2016;314:116-124. doi:10.1016/j. bbr.2016.08.001

20. Cheng SY, Zhao YD, Li J, et al. Plasma levels of glutamate during stroke is associated with development of post-stroke depression. Psychoneuroendocrinology. 2014;47:126-135. doi:10.1016/j.psyneuen. 2014.05.006
21. Williams JBW. A structured interview guide for the Hamilton depression rating scale. Arch Gen Psychiatry. 1988;45(8):742-747. doi:10.1001/archpsyc.1988.01800320058007

22. Zheng P, Chen J, Huang T, et al. A novel urinary metabolite signature for diagnosing major depressive disorder. J Proteome Res. 2013;12 (12):5904-5911. doi:10.1021/pr400939q

23. Hou L, Wei X, Zhuo Y, et al. GC-MS-based metabolomics approach to diagnose depression in hepatitis $\mathrm{B}$ virus-infected patients with middle or old age. Aging (Albany NY). 2018;10(9):2252. doi:10.18632/aging.101446

24. Guilloux JP, Douillard-Guilloux G, Kota R, et al. Molecular evidence for BDNF- and GABA-related dysfunctions in the amygdala of female subjects with major depression. Mol Psychiatry. 2012;17:1130-1142. doi:10.1038/mp.2011.113

25. Saxena SK, Ng TP, Koh G, Yong D, Fong NP. Is improvement in impaired cognition and depressive symptoms in post-stroke patients associated with recovery in activities of daily living? Acta Neurol Scand. 2007;115:339-346. doi:10.1111/j.1600-0404.2006.00751.x

26. Passaro A, Soavi C, Marusic U, et al. Computerized cognitive training and brain derived neurotrophic factor during bed rest: mechanisms to protect individual during acute stress. Aging (Albany NY). 2017;9(2):393-407. doi:10.18632/aging.101166

27. Sato Y, Chin Y, Kato T, et al. White matter activated glial cells produce BDNF in a stroke model of monkeys. Neurosci Res. 2009;65(1):71-78. doi:10.1016/j.neures.2009.05.010

28. Nomoto H, Baba H, Satomura E, et al. Serum brain derived neurotrophic factor levels and personality traits in patients with major depression. BMC Psychiatry. 2015;15:33. doi:10.1186/s12888-015-0413-1

29. Karege F, Perret G, Bondolfi G, et al. Decreased serum brain-derived neurotrophic factor levels in major depressed patients. Psychiatry Res. 2002;109(2):143-148.

30. Zhou C, Zhong J, Zou B, et al. Meta-analyses of comparative efficacy of antidepressant medications on peripheral BDNF concentration in patients with depression. PLoS One. 2017;12(2): $\mathrm{e} 0172270$. doi:10.1371/journal.pone.0172270

31. Shen $\mathrm{P}, \mathrm{Hu} \mathrm{Q}$, Dong $\mathrm{M}$, et al. Venlafaxine exerts antidepressant effects possibly by activating MAPK-ERK $1 / 2$ and P $13 \mathrm{~K}-\mathrm{AKT}$ pathways in the hippocampus. Behav Brain Res. 2017;335:63-70. doi:10.1016/j.bbr.2017.08.011

32. Hickie IB, Rogers NL. Novel melatonin-based therapies: potential advances in the treatment of major depression. Lancet. 2011;378 (9791):621-631. doi:10.1016/S0140-6736(11)60095-0

33. Zhao X, Cao F, Liu Q, et al. Behavioral, inflammatory and neurochemical disturbances in LPS and UCMS-induced mouse models of depression. Behav Brain Res. 2019;364:494-502. doi:10.1016/j. bbr.2017.05.064

34. Martinowich K, Lu B. Interaction between BDNF and serotonin: role in mood disorders. Neuropsychopharmacology. 2008;33(1):73-83. doi:10.1038/sj.npp.1301571

35. Chen J, Bai SJ, Li W, et al. Urinary biomarker panel for diagnosing patients with depression and anxiety disorders. Transl Psychiatry. 2018;8(1):192. doi:10.1038/s41398-018-0245-0

36. Chen J, Xie J, Zeng L, et al. Urinary metabolite signature in bipolar disorder patients during depressive episode. Aging (Albany NY). 2019;11(3):1008-1018. doi:10.18632/aging.101805 


\section{Publish your work in this journal}

Neuropsychiatric Disease and Treatment is an international, peerreviewed journal of clinical therapeutics and pharmacology focusing on concise rapid reporting of clinical or pre-clinical studies on a range of neuropsychiatric and neurological disorders. This journal is indexed on PubMed Central, the 'PsycINFO' database and CAS, and is the official journal of The International Neuropsychiatric Association (INA). The manuscript management system is completely online and includes a very quick and fair peer-review system, which is all easy to use. Visit http://www.dovepress.com/testimonials.php to read real quotes from published authors.

Submit your manuscript here: https://www.dovepress.com/neuropsychiatric-disease-and-treatment-journal 